\title{
OsRAR1 and OsSGT1 Physically Interact and Function in Rice Basal Disease Resistance
}

\author{
Yaling Wang, ${ }^{1}$ Mingjun Gao, ${ }^{1}$ Qun Li, ${ }^{1}$ Linyou Wang, ${ }^{2}$ Jianjun Wang, ${ }^{2}$ Jong-Seong Jeon, ${ }^{3} \mathrm{Na} \mathrm{Qu},{ }^{4}$ \\ Yuelin Zhang, ${ }^{4}$ and Zuhua $\mathrm{He}^{1}$ \\ ${ }^{1}$ National Key Laboratory of Plant Molecular Genetics, Institute of Plant Physiology \& Ecology, Shanghai Institutes \\ for Biological Sciences, Chinese Academy of Sciences, Shanghai, 200032, China; ${ }^{2}$ Zhejiang Academy of Agricultural \\ Sciences, Hangzhou 310021, China; ${ }^{3}$ Graduate School of Biotechnology \& Plant Metabolism Research Center, \\ Kyung Hee University, Yongini 451-701, Korea; ${ }^{4}$ National Institute of Biological Sciences, Beijing 102206, China
}

Submitted 22 August 2007. Accepted 25 October 2007.

\begin{abstract}
The RAR1 and SGT1 proteins function synergistically or antagonistically in plant innate immune responses. Here, we show that the rice orthologs OsRAR1 and OsSGT1 physically interact in vivo and in yeast. They displayed conserved roles in Arabidopsis disease resistance through ectopic expression in the Arabidopsis rarl and sgt1 mutants. Overexpression of OsRar1 and OsSGT1 in rice significantly increased basal resistance to a virulent bacterial blight Xanthomonas oryzae pv. oryzae PXO99 but not to another virulent strain DY89031, suggesting race-specificlike basal resistance conferred by $O$ sRarl and $O s S G T 1$. OsRar1-OE and OsSGT1-OE plants also enhanced resistance to all four virulent blast fungal Magnaporthe oryzae races. Overexpression of the OsSGT1-green fluorescent protein (GFP) fusion most likely caused a dominant negative phenotype which led to race-specific-like basal resistance. Transgenic plants overexpressing OsSGT1-GFP show enhanced resistance to DY89031 but decreased resistance to PXO99, implying that OsSGT1 might be the target of a component required for DY89031 virulence or OsSGT1GFP might stabilize weak resistance proteins against DY89031. Consistent with the hypothesis of the dominant negative regulation, we observed the reduced sensitivity to auxin of OsSGT1-GFP plants compared with the wild-type ones, and the curling-root phenotype in OsSGT1-OE plants. These results collectively suggest that OsRarl and OsSGT1 might be differentially required for rice basal disease resistance. Our current study also provides new insight into the roles of OsSGT1 in basal disease resistance.
\end{abstract}

Additional keywords: development, R-mediated resistance.

During their long-time co-evolution with microbes, plants have developed a sophisticated innate immune system that responds to infection through recognition of both general pathogen- and microbe-associated molecule patterns (PAMPs and MAMPs) by pattern recognition receptors (PRRs), and specific pathogen-encoded molecules (effectors) by disease resistance (R) proteins, resulting in defense responses (Abramovitch et al. 2006; Ausubel 2005; Chisholm et al. 2006; Dangl 2007).

Corresponding author: Z. He; E-mail: zhhe@ sibs.ac.cn; Telephone: +2154924121; Fax: +21-54924015.

* The $\boldsymbol{e}$-Xtra logo stands for "electronic extra" and indicates that supplemental material is available on line. Two additional figures and one additional table are published online.
Although it does not lead to strong disease resistance compared with R-mediated specific resistance, basal defense provides the first line of defense against pathogen attack. One such PRR is the well-characterized flagellin receptor kinase FLS2 that recognizes the highly conserved flg22 peptide, leading to immediate defense responses (Asai et al. 2002; Gomez-Gomez et al. 2001; Zipfel et al. 2004). Moreover, the recognition of flg22 induces FLS2 internalization associated with defense responses (Robatzek et al. 2006). In addition, some other important components involved in basal defense have been identified, of which EDS1 and its interacting protein PAD4 are required by both R resistance and basal defense (Falk et al. 1999; Feys et al. 2001; Jirage et al. 1999; Parker et al. 1996). Recently, a key regulator of R resistance, RIN4, also has been shown to modulate flg22 signaling (Kim et al. 2005). Collectively, both basal and specific immune signaling pathways appear to be continuum-linked.

As a key component involved in diverse $\mathrm{R}$ resistance, RAR1 is a novel eukaryotic zinc-binding protein. It is required for racespecific resistance to powdery mildew in barley (Halterman et al. 2001; Halterman and Wise 2004; Lahaye et al. 1998; Shen et al. 2003; Shirasu et al. 1999). Genetic screening also discovered that RAR1 is a key component for diverse R-mediated resistance in Arabidopsis (Holt et al. 2005; Muskett et al. 2002; Tornero et al. 2002). The Rarl gene also plays an important role in plant basal defense: mutations in Rarl enhance susceptibility to virulent pathogens in Arabidopsis and barley (Holt et al. 2005; Jarosch et al. 2005). Furthermore, AtRAR1 is a target of the Pseudomonas syringae effector AvrB that suppresses PAMP-triggered immunity (Shang et al. 2006). RAR1 contains two conserved CHORD domains required for physical interaction with the partner protein SGT1, which also plays an essential role in Mla12-mediated specific powdery mildew resistance in barely (Azevedo et al. 2002). Similarly, SGT1 protein is required by multiple R-mediated resistance and nonhost resistance in many species, including Arabidopsis, tobacco, wheat, pepper, and tomato (Austin et al. 2002; Leister et al. 2005; Liu et al. 2002; Peart et al. 2002; Schornack et al. 2004; Scofield et al. 2005; Tor et al. 2002; Zhang et al. 2004). It has been demonstrated that RAR1 and SGT1 directly interact with each other in planta, and the interaction of RAR1 and SGT1 is suggested to be essential for their functions in plant defense (Austin et al. 2002; Liu et al. 2002; Tor et al. 2002). RAR1 and SGT1 function synergistically in most $\mathrm{R}$ resistance. However, the Arabidopsis AtRAR1 and AtSGT1b play antagonistic roles in accumulation of some $\mathrm{R}$ proteins and in basal defense (Holt et al. 2005). A recent report further suggested that the antagonis- 
tic roles of AtRAR1 and AtSGT1b might result from the presence of two copies of SGT1, AtSGT1a and AtSGT1b, in Arabidopsis. AtSGT1a and AtSGT1b accumulate at different steady-state levels and diverse $\mathrm{R}$ proteins require different threshold of SGT1 to trigger effective resistance (Azevedo et al. 2006). Therefore, arguments arise on the precise mechanisms of RAR1 and SGT1 roles in plant disease resistance.

Silencing of the Rarl homolog chp in Caenorhabditis elegans by RNA interference (RNAi) resulted in semisterility and embryo lethality, indicating a role of RAR1 in development (Shirasu et al. 1999). In tobacco, both NbRAR1 and NbSGT1 are associated with the COP9 signalosome and the components of the Skp1/Cullin/F-box protein (SCF)-type E3 ubiquitin ligase complex, which is involved in protein degradation mediated by the COP9 signalosome (Liu et al. 2006). Furthermore, AtSGT1 is required for $\mathrm{SCF}^{\mathrm{TIR} 1}$-mediated degradation of Aux/indole-3acetic acid (IAA) proteins and is involved in the auxin-related processes, including auxin inhibition of root growth, lateral root development, hypocotyl elongation at high temperature, and apical dominance (Gray et al. 2003). Therefore, RAR1 and SGT1 link disease resistance to developmental processes. However, questions still remain as to the regulation of RAR1 and SGT1 in the cross-talk of plant defense and development.

Rice, as a monocot plant with known genome sequence, has been a model for studying the molecular mechanism of defense responses in cereal crops. The rice genome contains single-copy Rarl and one SGT1 homologous genes, OsRarl and
OsSGT1. Whether the two genes are involved in disease resistance remains unknown. Here, we report that the rice orthologs OsRAR1 and OsSGT1 physically interact with each other and function in basal disease resistance to rice bacterial leaf blight caused by Xanthomonas oryzae pv. oryzae and fungal blast caused by Magnaporthe oryzae in a race-specific-like basal resistance manner. We also present evidence that OsSGT1 functions in the cross-talk between defense and development, and that expression of the OsSGT1-green fluorescent protein (GFP) fusion might dominate or negatively regulate defense and root growth.

\section{RESULTS}

\section{OsRAR1 and OsSGT1 interact}

with each other in vivo and in yeast.

It was reported that the barely and Arabidopsis RAR1 and SGT1 proteins interact with each other via their CHORDII and CS domains (Azevedo et al. 2002). We first determined subcellular localization of the OsRAR1 and OsSGT1 proteins. Both DsRED-OsRAR1 and OsSGT1-GFP fusion proteins localize ubiquitously in the cytoplasm and nucleus (Fig. 1A and B). Furthermore, co-expression of OsSGT1-GFP and DsRED-OsRAR1 showed that they were co-localized (Fig. 1C), suggesting that they might physically interact with each other in vivo. We further performed a bimolecular fluorescence complementation (BiFC) assay in which the yellow fluorescent protein (YFP)
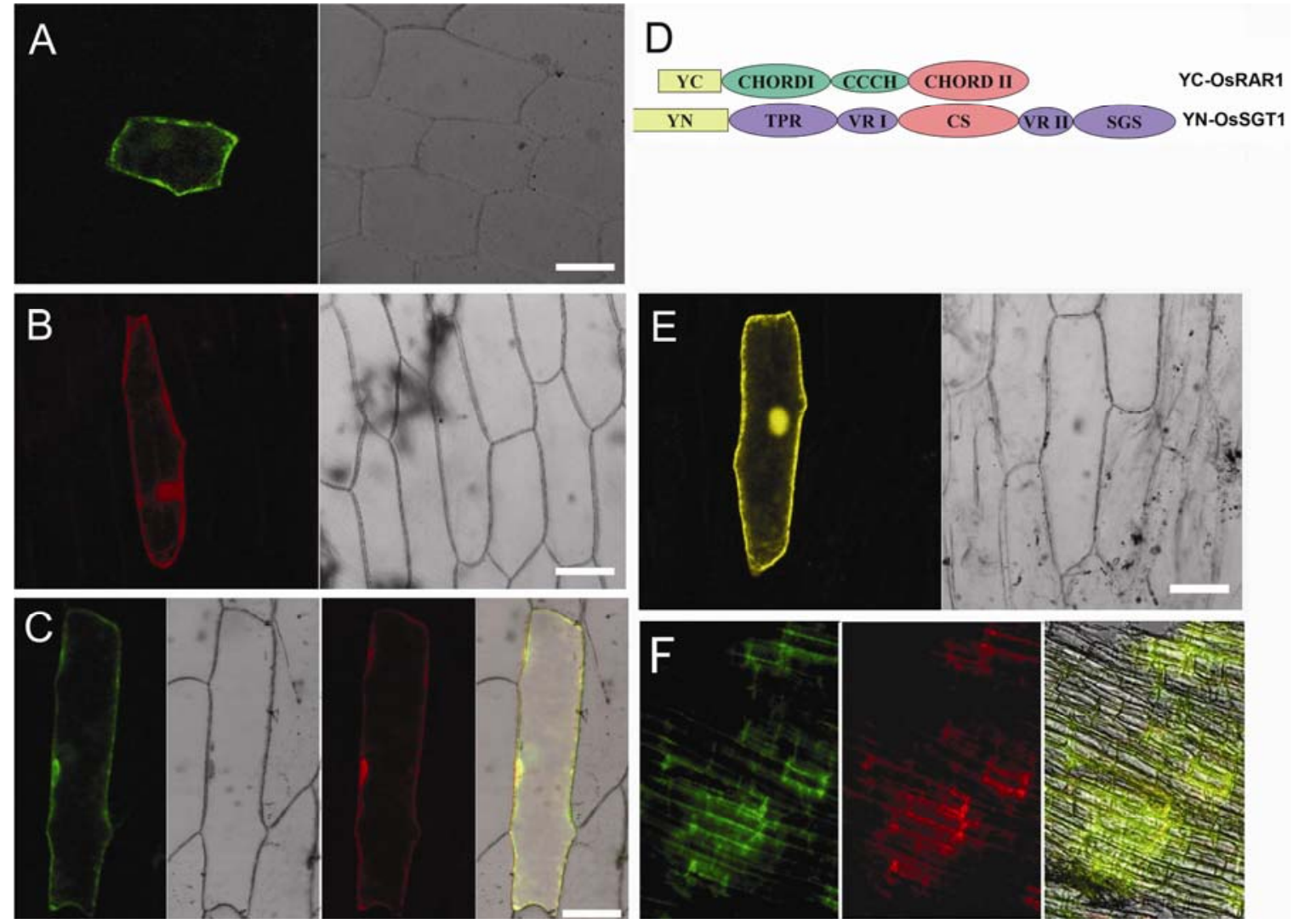

Fig. 1. Subcellular localization and physical interaction of OsRAR1 and OsSGT1 in onion epidermal cells. A, Cytoplasm and nuclear localization of the OsSGT1-green fluorescent protein (GFP) fusion protein. B, Cytoplasm and nuclear localization of the DsRED-OsRAR1 fusion protein. C, Co-localization of DsRED-OsRAR1 and OsSGT1-GFP in a single cell. Green and red fluorescence were detected in the same cell, together with bright field image of cells (AC). Merged image of green and red fluorescence generated yellow fluorescence image (left). D, Schematic illustration of the constructs for analysis of bimolecular fluorescence complementation (BiFC). The $\mathrm{N}$ and $\mathrm{C}$ termini of YFP were fused with OsSGT1 (YN-OsSGT1) and OsRAR1 (YC-OsRAR1), respectively. E, Fluorescence was restored when $Y N$-OsSGT1 and YC-OsRarl were co-expressed. Bright field image of cells was shown. F, Co-localization of OsSGT1-GFP and DsRED-OsRAR1 in the transgenic rice root. OsSGT1-GFP rice was crossed with DsRED-OsRar1 plant to obtain hybrid (F1) plants, Green (left) and red (middle) fluorescence were detected in the same cells. Merged image of green and red fluorescence generated yellow fluorescence image (right). The lengths of scale bars are given in $100 \mu \mathrm{m}$ in each panel. 
was split into two polypeptides (Fig. 1D), with the $\mathrm{N}$ terminus fused with OsSGT1 (YN-OsSGT1) and the C terminus with OsRAR1 (YC-OsRAR1). Co-expression of YN-OsSGT1 and YC-OsRAR1 reconstituted YFP fluorescence (Fig. 1E), whereas co-expression of YN-OsSGT1 and YC or YC-OsRAR1 and YN did not produce any fluorescence (data not shown). Collectively, we demonstrated that OsRAR1 and OsSGT1 physically interact with each other in vivo. We further examined the interacting domains of OsSGT1 and OsRAR1 using the yeast two-hybrid system. The result showed that OsRAR1 and OsSGT1 interact

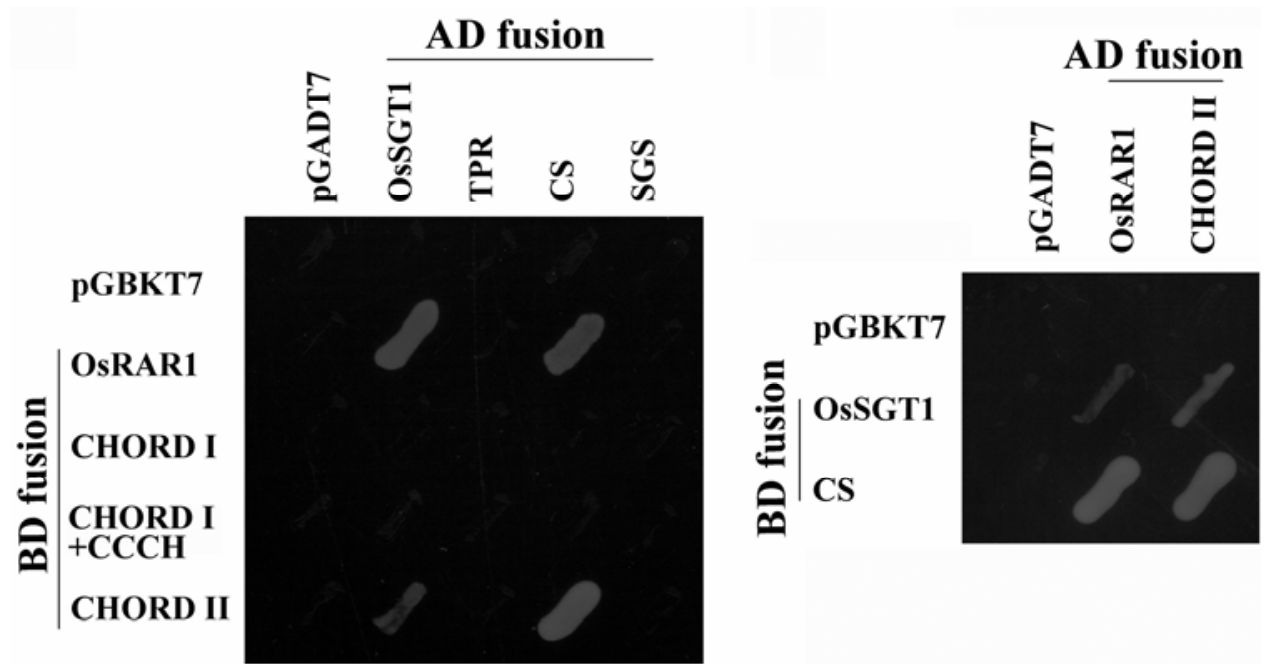

Fig. 2. Determination of interaction domains of OsRAR1 and OsSGT1 in yeast two-hybrid experiments. The full-length and partial coding sequences of OsSGT1 and OsRarl were inserted into the vectors pGAD-T7 (activator domain fusion, AD), pGBK-T7 (binding domain fusion, BD), or both, as indicated. These constructs were introduced into yeast AH109 cells with empty vectors as controls, and interactions were detected in high stringent screen medium with X- $\alpha$-gal according to the protocol for the GAL4 system.
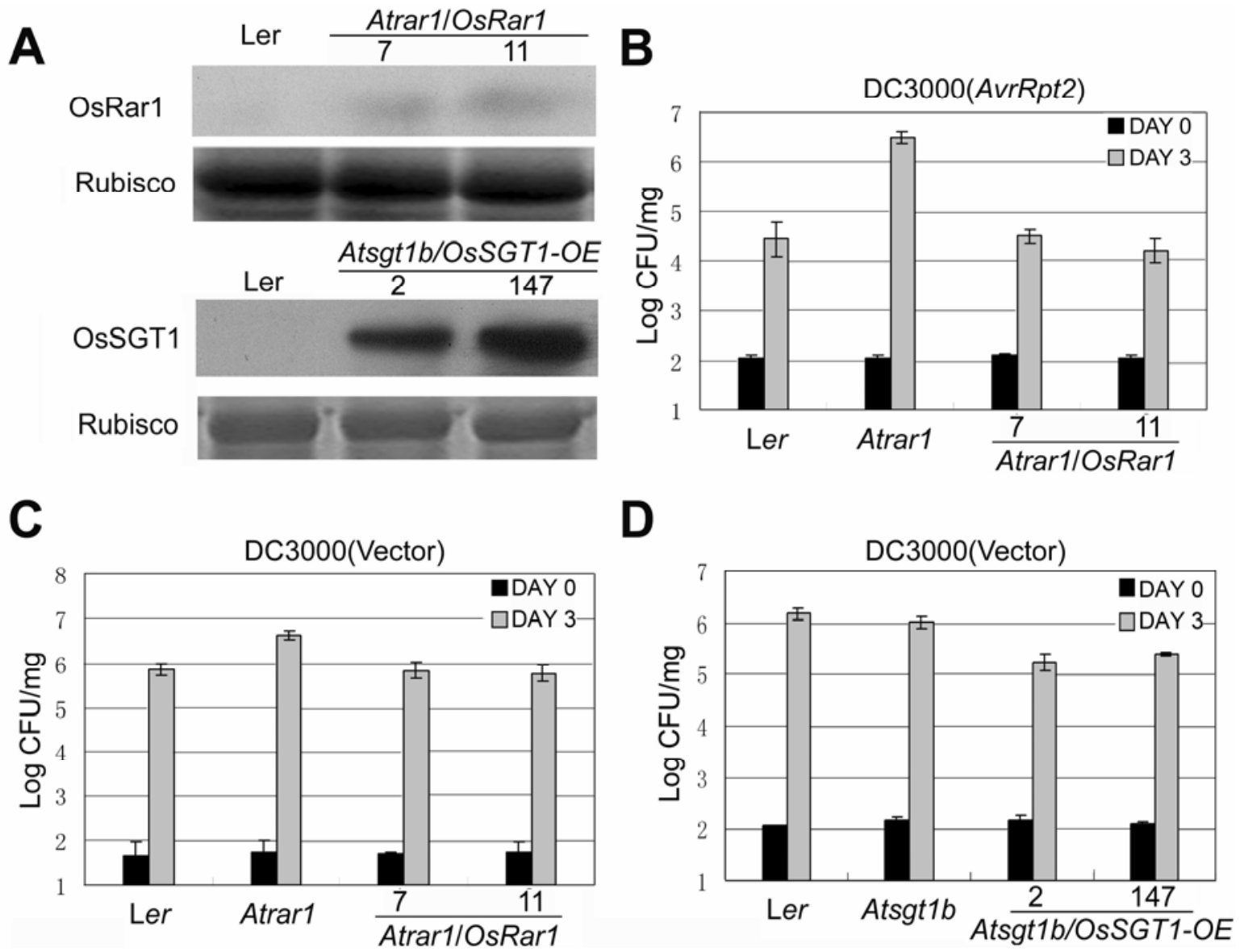

Fig. 3. Conserved function of OsRAR1 and OsSGT1 in Arabidopsis. A, Levels of OsRAR1 and OsSGT1 were detected by Western blot in the transformed Arabidopsis rarl and sgt1 mutants. Note that OsRAR1 accumulated to only a trace amount. B, Resistance gene-mediated resistance to DC3000 (AvrRpt2) was restored in the rarl mutant expressing the AtRarl-OsRarl chimera. C, Basal resistance to DC3000 was restored in the rarl mutant expressing the AtRar1-OsRarl chimera. D, Significantly $(P<0.05)$ enhanced basal resistance to DC3000 in the sgt $1 b$ mutant overexpressing OsSGT1. Each two representative lines of OsRarl and OsSGT1 transformants were shown. All experiments were repeated at least once with similar results. 
with each other via the CHORDII domain of OsRAR1 and the CS domain of OsSGT1 (Fig. 2), consistent with the previous observation (Azevedo et al. 2002).

\section{OsRarl and OsSGT1 exhibit conserved functions}

in Arabidopsis disease resistance.

Mutations in AtRarl lead to impairment of multiple R-mediated resistance and basal defense (Holt et al. 2005; Muskett et al. 2002; Tornero et al. 2002). High identity in sequences predicts that OsRarl and AtRarl could be functionally conserved. To test this hypothesis, we expressed OsRarl driven by the AtRarl promoter in the Atrarl-11 mutant, as indicated by protein levels (Fig. 3A). The introduction of OsRarl restored the RPS2mediated resistance of Atrarl to avirulent $P$. syringae pv. tomato DC3000 (AvrRpt2) to the same level of the wild-type Ler (Fig. 3B). In addition, OsRarl rescued basal disease resistance of Atrarl to virulent $P$. syringae pv. tomato DC3000 (Fig. 3C), indicating that OsRarl functions in basal defense as well.
Single mutation of neither AtSGTla nor AtSGTIb affects the basal defense of Arabidopsis. We constitutively expressed OsSGT1 (OsSGT1-OE) in the Atsgtlb-1 mutant (Fig. 3A). Inoculation with $P$. syringae pv. tomato DC3000 showed that the OsSGT1-OE lines statistically significantly decreased the growth of bacteria compared with the wild-type and Atsgtlb mutant (Fig. 3D). We also observed that the OsSGT1-OE lines restored the RPP5-mediated resistance to Peronospora parasitica race Noco2 (Table 1). Therefore, OsSGT1, like OsRarl, is also the rice ortholog and functions in both $\mathrm{R}$ and basal disease resistance in transgenic Arabidopsis. These results demonstrate that the rice ortholog OsRarl and OsSGT1 have conserved functions in Arabidopsis defense.

\section{OsRarl and OsSGT1 function}

in rice basal disease resistance to bacterial blight.

To determine roles of OsRarl and OsSGT1 in rice disease resistance, we generated transgenic rice constitutively expressing

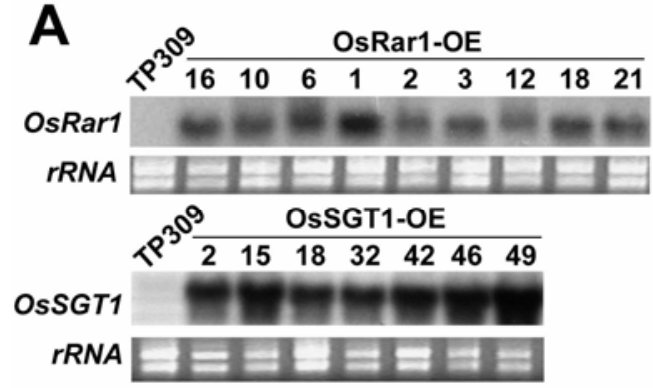

C

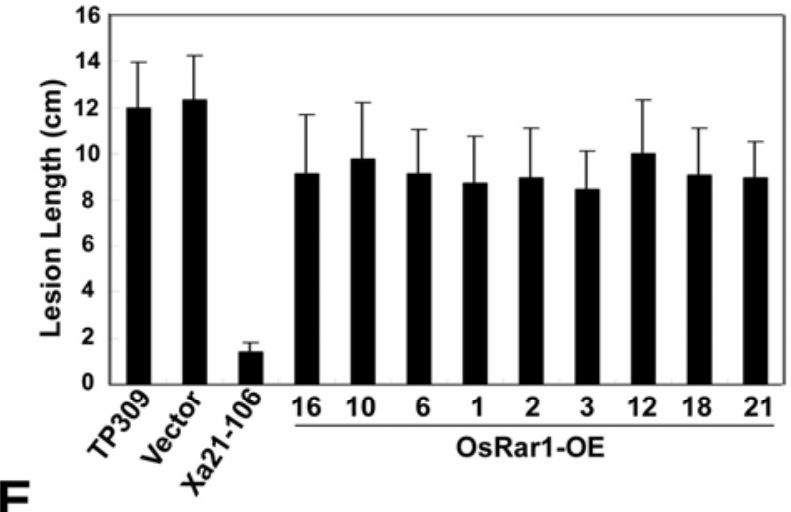

$\mathbf{E}$

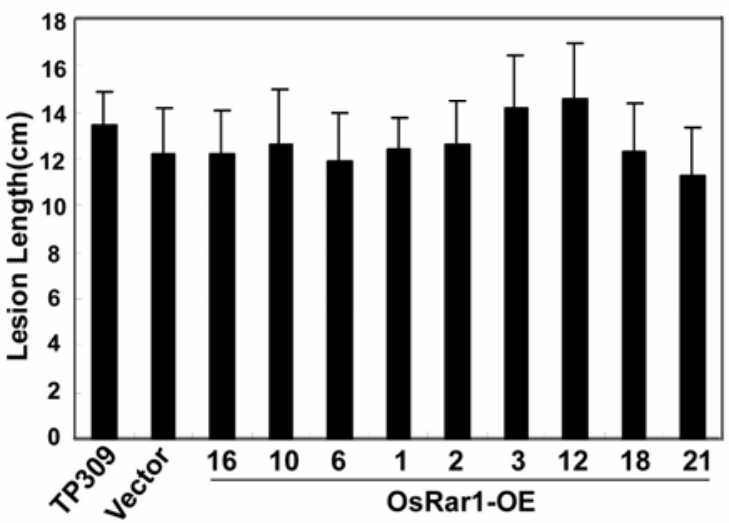

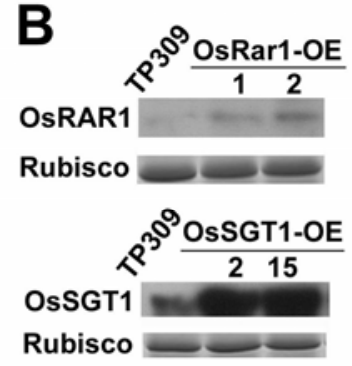

D
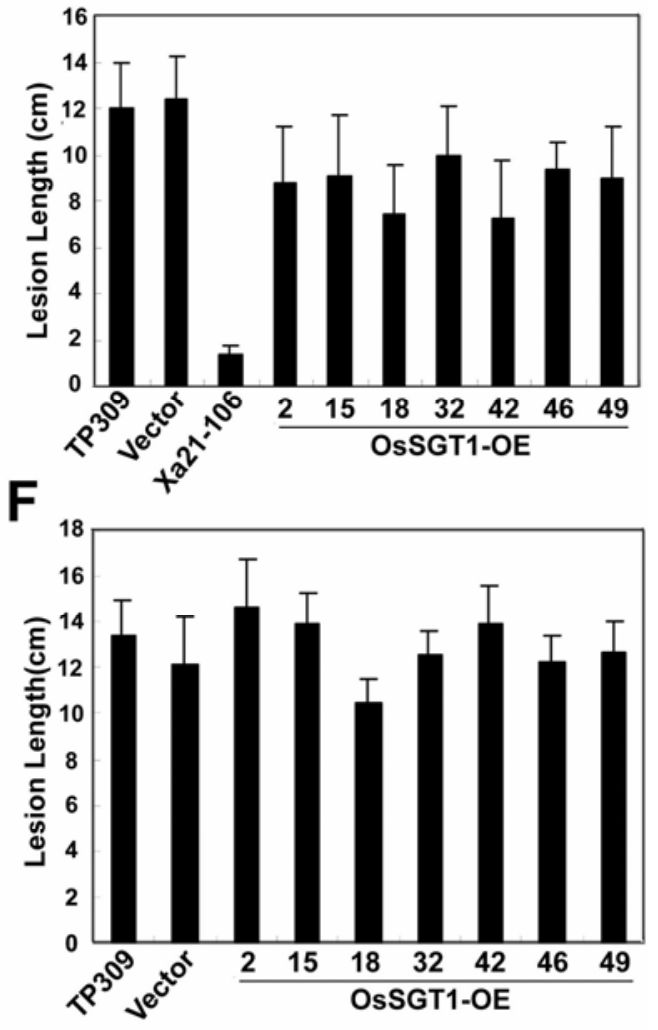

Fig. 4. Enhanced basal disease resistance to bacterial blight in OsRar1-OE and OsSGT1-OE plants. A, Northern blot detection of the OsRarl and OsSGT1 transcripts in transgenic plants. B, Levels of the OsRAR1 and OsSGT1 proteins detected by Western blot in each two representative transgenic lines, with Rubisco staining as a control. Note that both proteins were hardly detectable in the wild-type TP309. Disease resistance to virulent PXO99 was significantly enhanced $(P<0.05)$ in $\mathbf{C}$, OsRar1-OE and D, OsSGT1-OE plants compared with the wild-type and vector control. Basal disease resistance in E, OsRar1-OE and F, OsSGT1-OE plants to virulent Xanthomonas oryzae pv. oryzae DY89031. No reliable difference was observed in disease resistance in these plants compared with the wild-type and vector control. 
OsRarl and OsSGT1 (OsRar1-OE and OsSGT1-OE). RNA and Western blotting revealed that both OsRarl and OsSGT1 were overexpressed in the homozygous progeny plants at the transcript and protein levels (Fig. 4A and B). We inoculated transgenic plants with virulent $X$. oryzae pv. oryzae PXO99 and DY89031, in comparison with the wild-type TP309, vector control, and the transgenic TP309 line 106 (Xa21-106) harboring the resistance gene Xa21 to PXO99 but not DY89031 (Song et al. 1995; Yuan et al. 2007). All OsRar1-OE lines exhibited a statistically significant $(P<0.05)$ alleviation of disease symptom to PXO99 in comparison with the controls (Fig. 4C). Enhanced resistance to PXO99 was also observed for the OSSGT1 overexpressing lines (Fig. 4D). Intriguingly, none of the OsRar1-OE and OsSGT1-OE lines showed significant change in resistance to another virulent $X$. oryzae pv. oryzae strain, DY89031, with repeated experiments of two generations (Fig. 4E and F). These results suggest that OsRarl and OsSGT1 function in rice basal defense to bacterial blight in a race-specific-like manner (discussed below). Together with the data from transgenic Arabi-

Table 1. OsSGT1 restores RPP5-mediated resistance to Peronospora parasitica Noco2 in the Atsgt1b mutant

\begin{tabular}{lc}
\hline Line & Diseased plants/total plants \\
\hline Ler & $0 / 24$ \\
Atsgt1b-1 & $24 / 24$ \\
Atsgt1b/OSSGT1-OE 2 & $0 / 13$ \\
Atsgt1b/OsSGT1-OE 50 & $0 / 24$ \\
Atsgt1b/OsSGT1-OE 106 & $0 / 21$ \\
Atsgt1b/OsSGT1-OE 170 & $0 / 24$ \\
\hline
\end{tabular}

dopsis (Fig. 3D), we propose that SGT1 is also a positive regulator of plant basal defense.

\section{OsRarl and OsSGT1 function}

in rice basal disease resistance to blast fungus.

We further tested basal disease resistance of OsRar1-OE and OsSGT1-OE plants to blast fungus (M. oryzae). Unlike to $X$. oryzae pv. oryzae, both types of transgenic plants exhibited enhanced resistance to all the four tested virulent fungal races compared with the wild-type and vector transgenic controls (Fig. 5A and B), suggesting different requirements for OsRarl and $O S S G T 1$ in basal resistance to $X$. oryzae pv. oryzae and blast. Because there is no available Osrarl and Ossgt1 knockout mutants, we also extensively conducted RNA interference with both double-stranded RNA technology that has been successfully used to knockdown the $O S N P R 1$ gene in our previous study (Yuan et al. 2007) and antisense expression, but we did not manage to generate OsRarl and OsSGT1 knockdown or knockout plants (Supplemental Figure 1). It is possible that the regions of OsRAR1 and OsSGT1 chosen do not work well with the interference approach. It remains unknown what phenotypes, if any, are associated with knockout mutants of OsRarl and OsSGTI.

\section{OsSGT1-GFP fusion probably acts as a dominant negative form and confers a race-specific-like basal resistance.}

We generated rice plants (OsSGT1-GFP) constitutively expressing the OsSGT1-GFP fusion, in which both the OsSGT1GFP fusion protein and the endogenous OsSGT1 protein were
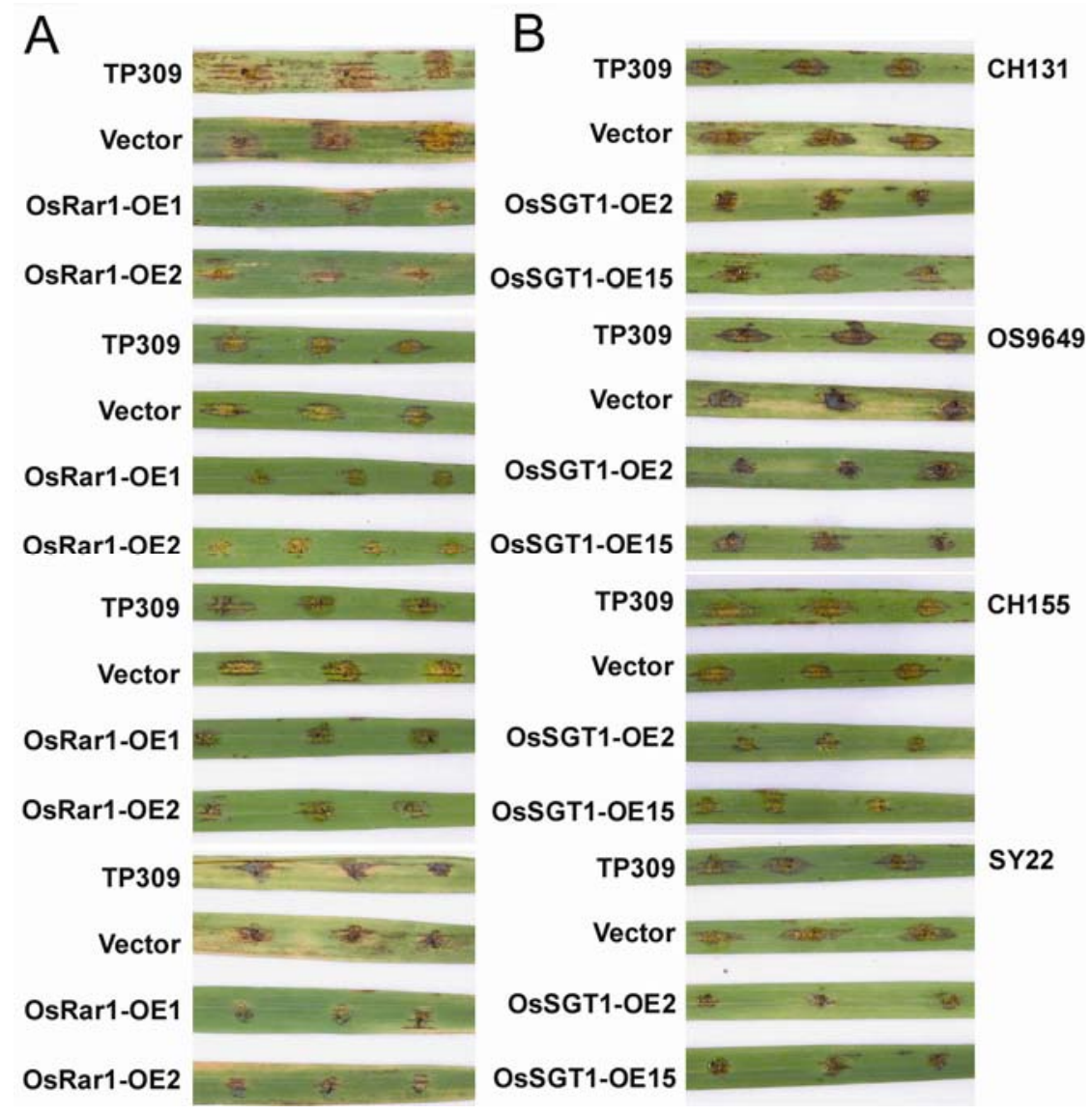

Fig. 5. Enhanced basal disease resistance to Magnaporthe oryzae in OsRar1-OE and OsSGT1-OE plants. Leaves were spot inoculated with spore suspensions of four blast races (CH131, OS9649, CH155, and SY22) and disease symptoms were recorded at 5 days postinoculation. Each two representative lines of A, OsRar1-OE and B, OsSGT1-OE plants were shown, together with the controls. Note that lesion development was inhibited in the transgenic plants compared with the wild-type and vector controls in independent inoculation experiments. 
produced (Fig. 6A, upper panel). OsSGT1-GFP accumulation was further confirmed by Western blotting with a commercial anti-GFP antibody (Fig. 6A, lower panel). The OsSGT1-GFP fusion also ubiquitously localized in the cytoplasm and nucleus of transgenic rice root cells (Fig. 1F). We observed that the basal disease resistance to PXO99 was significantly compromised in OsSGT1-GFP plants (Fig. 6B). Surprisingly, resistance to DY89031 was greatly increased in all lines to a level similar to $\mathrm{Xa21}$-triggered resistance to PXO99 with repeated experiments of two generations (Fig. 6C and D). Because TP309 does not contain known $R$ genes to either PXO99 or DY89031, we propose that OsSGT1-GFP most likely acts as a dominant negative form that inhibits the endogenous OsSGT1-mediated resistance to PXO99 but positively regulates resistance to DY89031. We also generated transgenic rice lines (DsREDOsRar1) constitutively expressing the DsRED-OsRarl fusion that express both the DsRED-OsRAR1 and endogenous OsRAR1 proteins (Fig. 7A). The DsRED-OsRAR1 fusion protein also was co-localized with OsSGT1-GFP fusion in the root cells of the transgenic hybrid plant (Fig. 1F), suggesting that the fusions do not change their physical interaction in the rice cell as in the onion cell. However, no changes were observed in resistance to both $X$. oryzae pv. oryzae strains in DsRED-OsRar1 plants (Fig. 7B and C). Whether the DsREDOsRAR1 fusion has lost its function remains to be investigated.
OsSGT1-OE plants did not display obvious morphological changes. Interestingly, their roots exhibited a dramatic curling pattern under culture conditions (Supplemental Figure 2), similar to the Arabidopsis Atmlo mutants (Sandra et al. 2007). We further measured root responses to auxins and found that OsSGT1-GFP plant roots were less sensitive to 2.4-dichlorophenoxyacetic acid (2,4-D) in comparison with wild-type roots, and no obvious difference was observed in responses to indole 3-acetic acid (IAA) between OsSGT1-GFP and wildtype plants. In light of SGT1 functions in the auxin-mediated development (Gray et al. 2003; Liu et al. 2002), these results indicate that OsSGT1 also is involved in the auxin-mediated pathways and that the SGT1-GFP fusion protein also might cause a dominant negative regulation on development mediated by the uncharacterized auxin pathway in rice.

\section{DISCUSSION}

Our study has demonstrated the conserved functions of OsRAR1 and OsSGT1 in rice immunity. The functions of RAR1 and SGT1 in defense responses likely are fulfilled through their physical interaction in a complex (Azevedo et al. 2002; Dodds and Schwechheimer 2002; Muskett and Parker 2003). We performed detailed experiments to visualize the molecular interaction between OsRAR1 and OsSGT1 (Fig. 1). We also showed that this interaction is via the CHORD II and CS do-
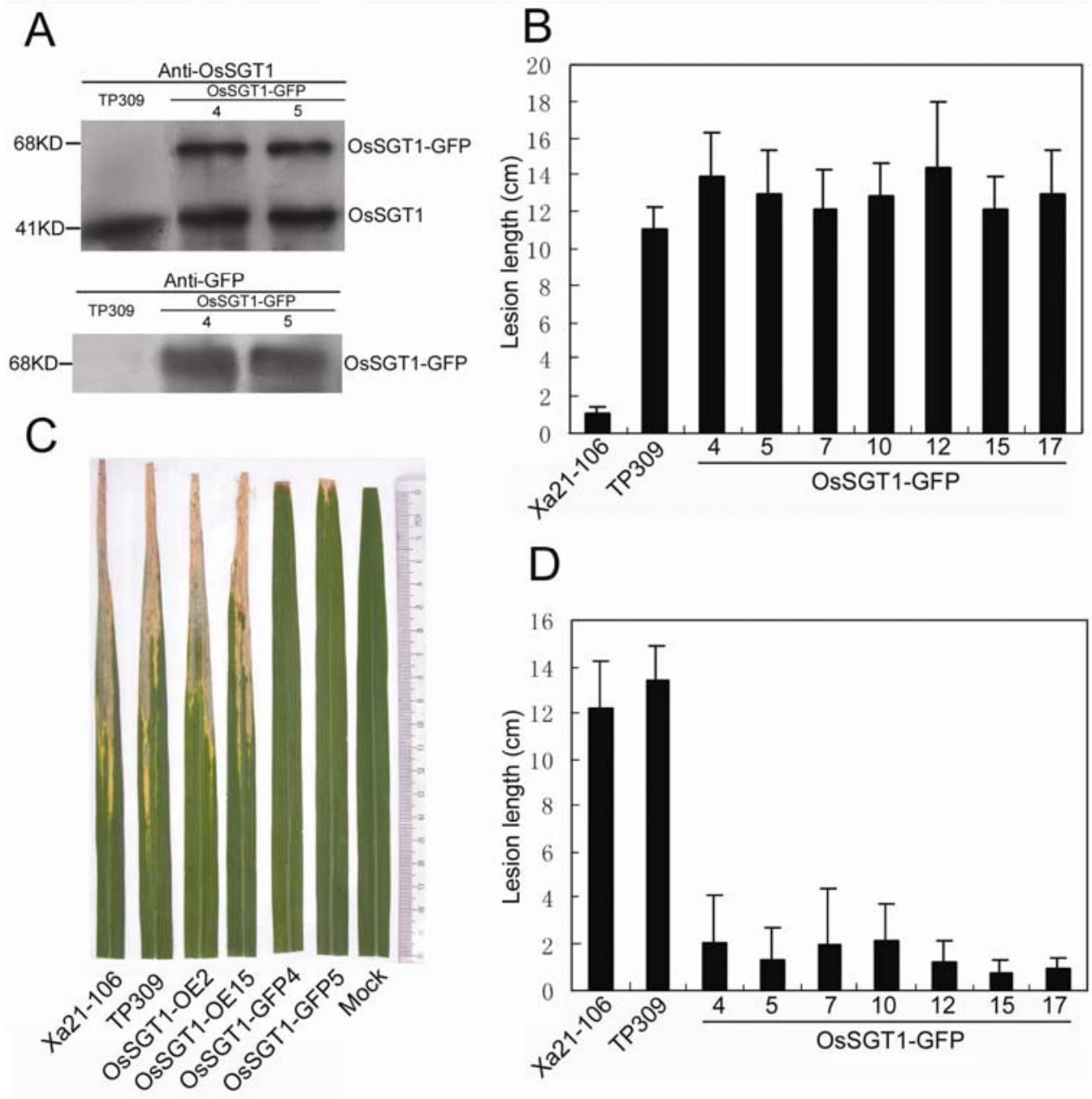

Fig. 6. Race-specific-like basal disease resistance in OsSGT1-green fluorescent protein (GFP) plants. A, Both the endogenous OsSGT1 protein and the OsSGT1-GFP fusion were expressed in two representative OsSGT1-GFP lines. B, Significant decreased $(P<0.05)$ resistance to virulent Xanthomonas oryzae pv. oryzae PXO99 in the OsSGT1-GFP lines compared with TP309, with Xa21-106 as the resistant control. C, High resistance to virulent X. oryzae pv. oryzae DY89031 in the OsSGT1-GFP lines, in comparison with TP309, Xa21-106, and OsSGT1-OE plants. D, Lesion lengths of OsSGT1-GFP, TP309, and Xa21-106 inoculated with X. oryzae pv. oryzae DY89031. 
mains of OsRAR1 and OsSGT1 (Fig. 2). Therefore, our study supports that the physical interaction is a common feature of RAR1 and SGT1 in diverse plant species.

RAR1 is required for basal defense in Arabidopsis and barley, probably through its contribution to $\mathrm{R}$ protein accumulation (Holt et al. 2005; Jarosch et al. 2005). We showed that overexpression of OsRarl and OsSGT1 restricted growth of one of the two virulent bacterial strains and all fungal races tested (Figs. 4 and 5). The difference in basal resistance of OsRar1-OE and OsSGT1-OE plants to $X$. oryzae pv. oryzae and $M$. oryzae probably resulted from distinct pathways by which rice responds to $X$. oryzae pv. oryzae and $M$. oryzae, as suggested by our microarray assay ( $\mathrm{Li}$ et al. 2006). Furthermore, we found that constitutively expressing OsSGT1 in an Arabidopsis sgtlb mutant also increased resistance to its virulent pathogen (Fig. 3D), indicating that $S G T 1$, like Rarl, also plays a positive role in plant basal defenses in addition to its involvement in $\mathrm{R}$ and nonhost disease resistance (Peart et al. 2002).

It is surprising that OsSGT1-OE and OsRar1-OE plants exhibited race-specific-like basal resistance to PXO99 and DY89031, suggesting that the two strains acquire divergent virulent components. Furthermore, when overexpressed as a GFP fusion, OsSGT1 appeared to cause a dominant negative phenotype that impaired basal disease resistance to PXO99 but induced a race-specific-like resistance to DY89031 (Fig. 6).
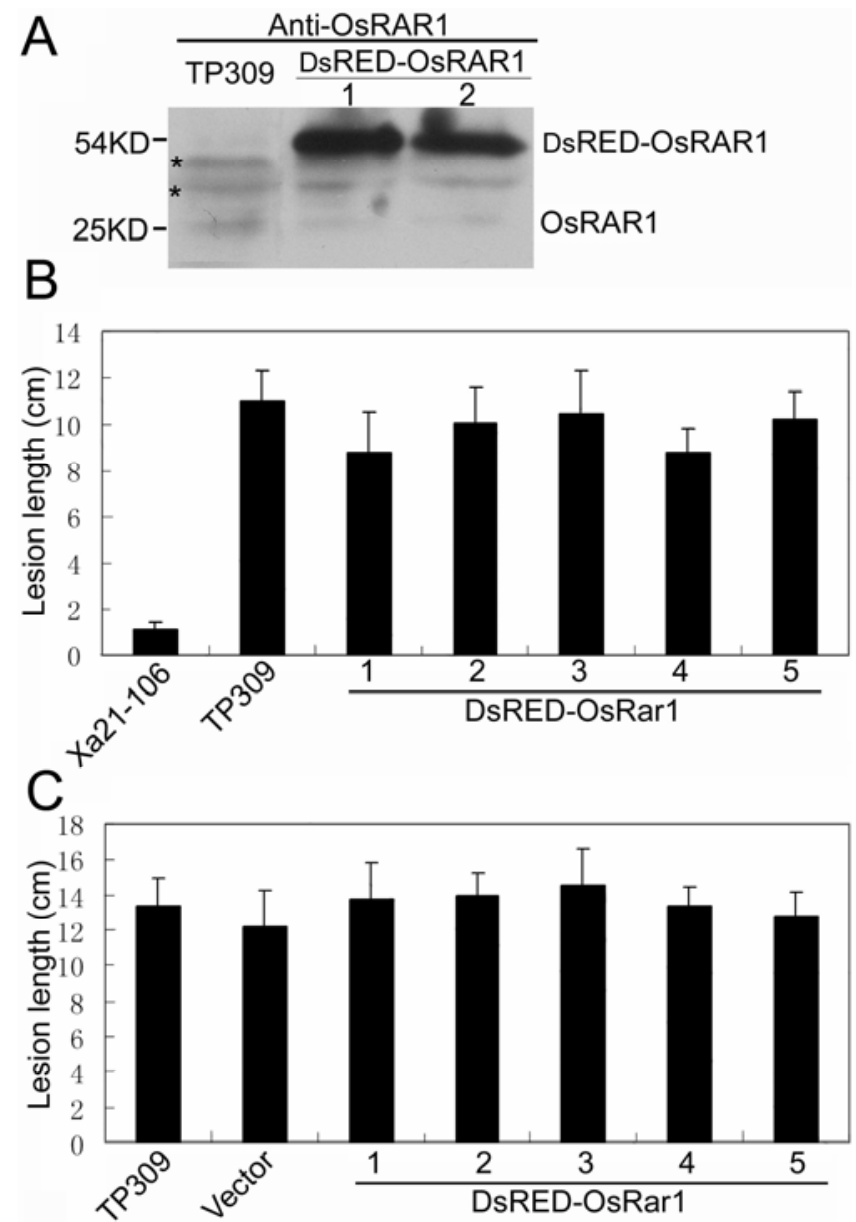

Fig. 7. Basal disease resistance in DsRED-OsRar1 plants. A, Both endogenous OsRAR1 and DsRED-OsRAR1 fusion were expressed in two representative DsRED-OsRar1 lines. Asterisks indicate nonspecific proteins detected by the OsRAR1 antibodies. B, Disease resistance to PXO99 in DsRED-OsRar1, TP309, and Xa21-106 plants. C, Disease resistance to DY89031 in DsRED-OsRar1, TP309, and vector control plants. No reliable difference was observed in disease resistance in these plants compared with the wild-type and vector control.
Similar dominant negative effects of GFP fusion proteins were observed in the GFP-phragmoplastin fusion protein on cell plate formation of tobacco BY-2 cells (Gu and Verma 1997) and the ASK1-GFP on Arabidopsis growth and fertility (Wang and Yang 2006).

These observations have not been reported in plants. The complicated phenotype may indicate different requirements for the SGT1 protein or its complex in basal defense to diverse bacterial strains, because SGT1 is required differently for diverse $R$ genes and nonhost resistance (Austin et al. 2002; Leister et al. 2005; Schornack et al. 2004; Scofield et al. 2005; Tor et al. 2002). Most likely, the OsSGT1-GFP fusion titrates out and the wild-type OsSGT1 (as well as OsRAR1) stabilizes weak R proteins that recognize uncharacterized effectors or pathogen determinants of PXO99. In this scenario, weak R proteins recognizing effectors of DY89031 may be different from those of PXO99 and the OsSGT1-GFP fusion might stabilize weak R proteins against DY89031. Alternatively, OsSGT1 may be the target of a component required for DY89031 virulence, and the OsSGT1-GFP fusion could have titrated out the component, similar to AtRAR1 as the target of the P. syringae AvrB (Shang et al. 2006). Consistent with our hypothesis, DY89031 contains mutations in the genes required for AvrXa21 activity to XA21 (Goes da Silva et al. 2004), which is proposed to be a secreted peptide acting as a quorum-sensing molecule with features of PAMP and Avr elicitors (Burdman et al. 2004; Lee et al. 2006). Further evidence that PAMPs derived from different bacterial strains induce strain-specific defense responses comes from the study that flagellin of $P$. avenae functions as a specific elicitor in rice (Che et al. 2000). A recent report also showed that sequence variation of flagellin among $X$. campestris pv. campestris strains affects FLS2-dependent pathogen recognition by Arabidopsis (Sun et al. 2006).

Our current study indicates that, although OsRAR1 and OsSGT1 physically interact with each other in planta and both function in basal disease resistance, mechanisms by which OsRAR1 and OsSGT1 regulate basal defense might be different, because the DsRED-OsRAR1 fusion protein appeared to not alter resistance to $X$. oryzae pv. oryzae (Fig. 7). Consistent with this idea, RAR1 and SGT1 in other plant species also function differently or even antagonistically in some $R$-genemediated resistance (Holt et al. 2005; Leister et al 2005). It will be interesting to further investigate the mechanism of finetuning the RAR1-SGT1 interaction and additional components in the RAR1-SGT1 complex as previously proposed (Dodds and Schwechheimer 2002; Muskett and Parker 2003). OsSGT1 loss-of-function data is not available currently; therefore, the dominant negative-like phenotype of OsSGT1-GFP needs to be further characterized genetically.

The OsSGT1-GFP fusion function is supported by the developmental phenotype. Because SGT1 has been shown to act cooperatively with the $\mathrm{SCF}^{\mathrm{TIR} 1}$-mediated auxin pathway and the COP9 signalosome (Gray et al. 2003; Liu et al. 2006), our data point strongly to the possibility that OsSGT1 and OsSGT1-GFP differently regulate the rice COP9 signalosome and $\mathrm{SCF}^{\mathrm{TIR} 1}$ complex that interactively play key roles in plant development through ubiquitin-proteasome-mediated protein degradation (Wei and Deng 2003). Due to lacking functional information of the rice COP9 and $\mathrm{SCF}^{\mathrm{TIR} 1}$ complexes (Yamamoto et al. 2003), we currently do not know how the OsSGT1-GFP-mediated dominant negative regulation occurs. Our preliminary data showed that OsSGT1GFP roots were less sensitive to $2,4-\mathrm{D}$ but not to IAA in comparison with wild-type roots. It is possible that the OsSGT1-GFP fusion might affect a particular auxin transport system differentially required for 2,4-D and IAA responses, as previously reported (Zhuang et al. 2006). Interestingly, the 
curling root phenotype also is associated with the Atmlo mutants (Sandra et al. 2007). Overexpression of OsSGT1 leads to this phenotype in rice, probably through regulating the interaction between SGT1 and RAR1, because the latter has been shown to be associated with the MLO function and rice contains a functional MLO ortholog (Elliott et al. 2002). The detailed proteomic and genetic analysis, and the identification of the SGT1-interacting components that is required for DY89031 virulence and probably for $\mathrm{COP} 9 / \mathrm{SCF}^{\mathrm{TIR} 1}$-mediated development as well, would provide a clue for the OsSGT1mediated phenotypes.

\section{MATERIALS AND METHODS}

\section{Gene cloning and plasmid construction.}

Using publicized genome sequences of rice, we isolated the 1.2-kb full-length OsSGT1 cDNA (GenBank accession number AF192467) from a rice cDNA library, which was inserted into $K p n I$ and BamHI sites of the binary vector 35S-C1301 (provided by P. Ronald) to form the OsSGT1 overexpression construct OsSGT1-OE. The full-length cDNA of OsRarl (GenBank accession number C28356) was obtained from the National Institute of Agrobiological Sciences (Tsukuba, Japan) and was inserted into ApaI and SacI sites of 35S-C1301 to form the overexpression construct OsRarl-OE. For complementation of the Arabidopsis rarl mutant, the OsRarl cDNA was placed under the promoter of AtRarl to generate the AtRarl-OsRarl chimera that was inserted into pCAMBIA1301 for Arabidopsis transformation. The construct OsSGT1-OE also was transformed into the Atsgtlb mutant. For RNAi, a 600-bp OsRarl or OsSGT11 cDNA fragment was reversely inserted into 35SC1301 to generate antisense expression plasmid, or was inserted into the double-stranded RNA interference vector $1300 \mathrm{~S}$ to form RNAi plasmid (Yuan et al. 2007).

\section{Rice and Arabidopsis transformation.}

Rice and Arabidopsis transformation was performed with the Agrobacterium-mediated method. The rice cv. Taipei 309 (TP309) was used as recipient to produce more than 20 independent transgenic lines for each construct. All transformants were confirmed by genomic DNA polymerase chain reaction and $\beta$-glucuronidase staining, and the homozygous progenies (T2 to T4) were used for all assays. The Arabidopsis mutants rarl-11 and sgtlb-1 (Austin et al. 2002; Muskett et al. 2002) were transformed with AtRarR1-OsRarl and OsSGT1-OE for complementation experiments. Transformants were screened with hygromycin and homozygous progeny lines were used for pathogen inoculation.

\section{Pathogen inoculation.}

Seven-week-old plants of independent transgenic lines and controls were inoculated with $X$. oryzae pv. oryzae races P6 (strain PXO99) and K1 (strain DY89031) using the leaf clip method (Song et al. 1995). After 2 weeks, lesion lengths were recorded. For blast inoculation, four virulent races (OS9649, Sy22, CH155, and CH131) and a spot-inoculation method were applied (Xiong and Yang 2003). Leaves were pierced with a needle, and a droplet of spore suspension (10 $\mu$ l containing approximately 50 spores) was inoculated on the punctured sites. Inoculated leaves were kept in a growth chamber with light intensity of $240 \mu \mathrm{m} \mathrm{m}^{-2} \mathrm{~s}^{-1}$ at $26^{\circ} \mathrm{C}$, and disease symptoms were recorded at 5 and 7 days postinoculation. $P$. syringae inoculation on Arabidopsis was performed with the syringe injection method. The bacterial pathogen growth curve was measured with independent assays. Arabidopsis inoculation with Peronospora parasitica race Noco 2 was performed as previously described (Bowling et al. 1997).
Subcellular localization and BiFC analysis.

The full-length coding regions of OsSGT1 and OsRarl were in-frame fused with $m G F P$ (GenBank accession number U87973) and DsRED (GenBank accession number AF168419) in 35S-C1301 with appropriate sites (Supplemental Table 1). The OsSGT1-GFP and DsRED-OsRar1 fusions were expressed transiently in onion epidermal cells by particle bombardment. Transgenic rice also was generated with the fusion constructs. For analysis of BiFC of OsSGT1 and OsRAR1 interaction, the fragments corresponding to the N (YN1-155) and C (YC156239) termini of YFP (GenBank accession number AF292557) were fused in-frame with OsSGT1 and OsRarl, respectively, to generate BiFC expression constructs YN-OsSGT1 and YCOsRar1 in 35S-C1301 with appropriated sites, which were cointroduced into onion epidermal cells by particle bombardment. Fluorescent images were observed with a confocol laser microscopy (Zeiss LSM510).

\section{Yeast two-hybrid assay.}

The full-length and partial coding regions of OSSGT1 and OsRarl were recombined in frame into pGAD-T7 and pGBKT7 (Clontech, Palo Alto, CA, U.S.A.), respectively, to construct yeast two-hybrid pairs with appropriate restriction enzyme sites. Yeast transformation and protein interaction were performed according to the manufacturer's protocol.

\section{RNA preparation and Northern blotting.}

Total RNA was prepared from leaf tissues using TRIzol reagent according to the manufacturer's instructions (Gibco BRL, Gaithersburg, MD, U.S.A.). RNA samples (20 $\mu \mathrm{g})$ were separated on a $1 \%$ formaldehyde-agarose gel, and then blotted onto Hybond-N membranes (Amersham, Tokyo). The 1.1-kb OsRar and a 1.2-kb OsSGT1 fragments were labeled with [ $\alpha-$ $\left.{ }^{32} \mathrm{P}\right] \mathrm{dCTP}$ using a random primer labeling kit (Takara) for hybridization and autoradiography.

\section{Protein analysis.}

The full-length coding regions of OsRarl and OsSGT1 were ligated into pET-32a to produce the fusion proteins in the Escherichia coli BL21(DE3). Antibodies (antiserum) against the OsRAR1 and OsSGT1 fusion proteins were raised with standard procedure. Western blotting was performed using the SuperSignal West chemiluminescence kit according to the manufacturer's protocol (Pierce, Rockford, IL, U.S.A.).

\section{Auxin responses.}

For analysis of auxin responses, 2,4-D and IAA of different concentrations were added to $1 / 2$ Murashige Skoog medium. Sterilized rice seed were germinated on the media at $60 \mu \mathrm{m}$ $\mathrm{m}^{-2} \mathrm{~s}^{-1}$ light intensity, $26^{\circ} \mathrm{C}$, and day and night length of 14 and $10 \mathrm{~h}$, respectively. Root length was measured at day 7 after germination.

\section{ACKNOWLEDGMENTS}

We thank J. Zhou and W. Tang for critical reading of the manuscript, X. Dong for helpful comments, and J. Parker for providing the rarl and sgtl mutants. The study was supported by grants from the Chinese Ministry of Science and Technology (2006CB101900 and 2006AA10A102), National Natural Science Foundation of China (30421001), and Chinese Academy of Sciences (KSCX2-YW-N-005) to Z. He., and a grant from the Crop Functional Genomic Center (CG1422), the 21 Century Frontier Program to J.-S. Jeon.

\section{LITERATURE CITED}

Abramovitch, R. B., Anderson, J. C., and Martin, G. B. 2006. Bacterial elicitation and evasion of plant innate immunity. Nat. Rev. Mol. Cell. Biol. 7:601-611. 
Asai, T., Tena, G., Plotnikova, J., Willmann, M. R., Chiu, W. L., GomezGomez, L., Boller, T., Ausubel, F. M., and Sheen, J. 2002. MAP kinase signalling cascade in Arabidopsis innate immunity. Nature 415:977983

Austin, M. J., Muskett, P., Kahn, K., Feys, B. J., Jones, J. D., and Parker, J E. 2002. Regulatory role of $S G T 1$ in early $R$ gene-mediated plant defenses. Science 295:2077-2080.

Ausubel, F. M. 2005. Are innate immune signaling pathways in plants and animals conserved? Nat. Immunol. 6:973-979.

Azevedo, C., Sadanom, A., Kitagawa, K., Freialdenhoven, A., Shirasu, K. and Schulze-Lefert, P. 2002. The RAR1 interactor SGT1, an essential component of $R$ gene-triggered disease resistance. Science 295:20732076

Azevedo, C., Betsuyaku, S., Peart, J., Takahashi, A., Noel, L., Sadanom, A., Casais, C., Parker, J., and Shirasu, K. 2006. Role of SGT1 in resistance protein accumulation in plant immunity. EMBO (Eur. Mol. Biol. Organ.) J. 25:2007-2016.

Bowling, S. A., Clarke, J. D., Liu. Y., Klessig, D. F., and Dong, X. 1997. The cpr5 mutant of Arabidopsis expresses both NPR1-dependent and NPR1-independent resistance. Plant Cell 9:1573-1584.

Burdman, S., Shen, Y., Lee, S. W., Xue, Q., and Ronald, P. 2004 RaxH/RaxR: a two-component regulatory system in Xanthomonas oryzae pv. oryzae required for AvrXa21 activity. Mol. Plant-Microbe Interact. 17:602-612.

Che, F. S., Nakajima, Y., Tanaka, N., Iwano, M., Yoshida, T., Takayama, S., Kadota, I., and Isogai, A. 2000. Flagellin from an incompatible strain of Pseudomonas avenae induces a resistance response in cultured rice cells. J. Biol. Chem. 275:32347-32356.

Chisholm, S. T., Coaker, G., Day, B., and Staskawicz, B. J. 2006. Hostmicrobe interactions: shaping the evolution of the plant immune response. Cell 124:803-814.

Dangl, J. L. 2007. Nibbling at the plant cell nucleus. Science 5815:1088 1089

Dodds, P. N., and Schwechheimer, C. 2002. A breakdown in defense signaling. Plant Cell (Suppl.)14:S5-8.

Elliott, C., Zhou, F., Spielmeyer, W., Panstruga, R., and Schulze-Lefert, P. 2002. Functional conservation of wheat and rice Mlo orthologs in defense modulation to the powdery mildew fungus. Mol. Plant-Microbe Interact. 15:1069-1077

Falk, A., Feys, B. J., Frost, L. N., Jones, J. D., Daniels, M. J., and Parker, J. E. 1999. EDS1, an essential component of $R$ gene-mediated disease resistance in Arabidopsis has homology to eukaryotic lipases. Proc. Natl. Acad. Sci. U.S.A. 96:3292-3297.

Feys, B. J., Moisan, L. J., Newman, M. A., and Parker, J. E. 2001. Direct interaction between the Arabidopsis disease resistance signaling proteins, EDS1 and PAD4. EMBO (Eur. Mol. Biol. Organ.) J. 20:54005411.

Goes da Silva, F., Shen, Y., Dardick, C., Burdman, S., Yadav, R., Sharma, P., and Ronald, P. 2004. Bacterial genes involved in type I secretion and sulfation are required to elicit the rice $\mathrm{Xa21}$-mediated innate immune response. Mol. Plant-Microbe Interact. 17:593-601

Gomez-Gomez, L., Bauer, Z., and Boller, T. 2001. Both the extracellular leucine-rich repeat domain and the kinase activity of FSL2 are required for flagellin binding and signaling in Arabidopsis. Plant Cell 13:11551163

Gray, W. M., Muskett, P. R., Chuang, H. W., and Parker, J. E. 2003. Arabidopsis SGT1b is required for SCF(TIR1)-mediated auxin response. Plant Cell 15:1310-1319.

Gu, X., and Verma, D. P. 1997. Dynamics of phragmoplastin in living cells during cell plate formation and uncoupling of cell elongation from the plane of cell division. Plant Cell 9:157-169.

Halterman, D., Zhou, F., Wei, F., Wise, R. P., and Schulze-Lefert, P. 2001. The MLA6 coiled-coil, NBS-LRR protein confers AvrMla6-dependent resistance specificity to Blumeria graminis f. sp. hordei in barley and wheat. Plant J. 25:335-348.

Halterman, D. A., and Wise, R. P. 2004. A single-amino acid substitution in the sixth leucine-rich repeat of barley MLA6 and MLA13 alleviates dependence on RAR1 for disease resistance signaling. Plant J. 38:215226

Holt, B. F., Belkhadir, Y., and Dangl, J. L. 2005. Antagonistic control of disease resistance protein stability in the plant immune system. Science 309:929-932.

Jarosch, B., Collins, N. C., Zellerhoff, N., and Schaffrath, U. 2005 RAR1, ROR1, and the actin cytoskeleton contribute to basal resistance to Magnaporthe grisea in barley. Mol. Plant-Microbe Interact. 18:397-404

Jirage, D., Tootle, T. L., Reuber, T. L., Frost, L. N., Feys, B. J., Parker, J. E., Ausubel, F. M., and Glazebrook, J. 1999. Arabidopsis thaliana PAD4 encodes a lipase-like gene that is important for salicylic acid signaling. Proc. Natl. Acad. Sci. U.S.A. 96:13583-13588.
Kim, M. G., da Cunha, L., McFall, A. J., Belkhadir, Y., DebRoy, S., Dangl, J. L., and Mackey, D. 2005. Two Pseudomonas syringae type III effectors inhibit RIN4-regulated basal defense in Arabidopsis. Cell 121:749759.

Lahaye, T., Shirasu, K., and Schulze-Lefert, P. 1998. Chromosome landing at the barley Rarl locus. Mol. Gen. Genet. 260:92-101.

Lee, S. W., Han, S. W., Bartley. L. E., and Ronald, P. C. 2006. Unique characteristics of Xanthomonas oryzae pv. oryzae AvrXa21 and implications for plant innate immunity. Proc. Natl. Acad. Sci. U.S.A. 103:18395-18400.

Leister, R. T., Dahlbeck, D., Day, B., Li, Y., Chesnokova, O., and Staskawicz, B. J. 2005. Molecular genetic evidence for the role of SGT1 in the intramolecular complementation of Bs2 protein activity in Nicotiana benthamiana. Plant Cell 17:1268-1278.

Li, Q., Chen, F., Sun, L., Zhang, Z., Yang, Y., and He, Z. 2006. Expression profiling of rice genes in early defense responses to blast and bacterial blight pathogens using cDNA microarray. Physiol. Mol. Plant Pathol. 68:51-60.

Liu, Y., Schiff, M., Serino, G., Deng, X. W., and Dinesh-Kumar, S. P. 2002. Role of SCF ubiquitin-ligase and the COP9 signalosome in the $N$ gene-mediated resistance response to Tobacco mosaic virus. Plant Cell 14:1483-1496.

Muskett, P., and Parker, J. 2003. Role of SGT1 in the regulation of plant $R$ gene signalling. Microbes Infect. 5:969-976.

Muskett, P. R., Kahn, K., Austin, M. J., Moisan, L. J., Sadanom, A. Shirasu, K., Jones, J. D., and Parker, J. E. 2002. Arabidopsis RAR1 exerts rate-limiting control of $\mathrm{R}$ gene-mediated defenses against multiple pathogens. Plant Cell 14:979-992.

Parker, J. E., Holub, E. B., Frost, L. N., Falk, A., Gunn, N. D., and Daniels, M. J. 1996. Characterization of eds1, a mutation in Arabidopsis suppressing resistance to Peronospora parasitica specified by several different RPP genes. Plant Cell 8:2033-2046.

Peart, J. R., Lu, R., Sadanandom, A., Malcuit, I., Moffett, P., Brice, D. C., Schauser, L., Jaggard, D. A., Xiao, S., Coleman, M. J., Dow, M., Jones, J. D., Shirasu, K., and Baulcombe, D. C. 2002. Ubiquitin ligase-associated protein SGT1 is required for host and nonhost disease resistance in plants. Proc. Natl. Acad. Sci. U.S.A. 99:10865-10869.

Robatzek, S., Chinchilla, D., and Boller, T. 2006. Ligand-induced endocytosis of the pattern recognition receptor FLS2 in Arabidopsis. Genes Dev. 20:537-542.

Sandra, N., Jones, A. M., Chen, Z., and Panstruga, R. 2007. In: 18th Int Conf. Arabidopsis Res. Beijing.

Schornack, S., Ballvora, A., Gurlebeck, D., Peart, J., Baulcombe, D., Ganal, M., Baker, B., Bonas, U., and Lahaye, T. 2004. The tomato resistance protein Bs4 is a predicted non-nuclear TIR-NB-LRR protein that mediates defense responses to severely truncated derivatives of AvrBs4 and overexpressed AvrBs3. Plant J. 37:46-60.

Scofield, S. R., Huang, L., Brt, A. S., and Gill, B. S. 2005. Development of a virus-induced gene-silencing system for hexaploid wheat and its use in functional analysis of the $\mathrm{Lr} 21$-mediated leaf rust resistance pathway. Plant Physiol. 138:2165-2173.

Shang, Y., Li, X., Cui, H., He, P., Thilmony, R., Chintamanani, S., ZwieslerVollick, J., Gopalan, S., Tang, X., and Zhou, J. 2006. RAR1, a central player in plant immunity, is targeted by Pseudomonas syringae effector AvrB. Proc. Natl. Acad. Sci. U.S.A. 103:19200-19205.

Shen, Q. H., Zhou, F., Bieri, S., Haizel, T., Shirasu, K., and SchulzeLefert, P. 2003. Recognition specificity and RAR1/SGT1 dependence in barley Mla disease resistance genes to the powdery mildew fungus. Plant Cell 15:732-744.

Shirasu, K., Lahaye, T., Tan, M. W., Zhou, F., Azevedo, C., and SchulzeLefert, P. 1999. A novel class of eukaryotic zinc-binding proteins is required for disease resistance signaling in barley and development in $C$. elegans. Cell 99:355-366.

Song, W. Y., Wang, G. L., Chen, L. L., Kim, H. S., Pi, L. Y., Holsten, T., Gardner, J., Wang, B., Zhai, W. X., Zhu, L. H., Fauquet, C., and Ronald, P. 1995. A receptor kinase-like protein encoded by the rice disease resistance gene, Xa21. Science 270:1804-1806.

Sun, W., Dunning, F. M., Pfund, C., Weingarten, R., and Bent, A. F. 2006. Within-species flagellin polymorphism in Xanthomonas campestris pv. campestris and its impact on elicitation of Arabidopsis FLAGELLIN SENSING2-dependent defenses. Plant Cell 18:764-779.

Tor, M., Gordon, P., Cuzick, A., Eulgem, T., Sinapidou, E., Mert-Turk, F., Can, C., Dangl, J. L., and Holub, E. B. 2002. Arabidopsis SGT1b is required for defense signaling conferred by several downy mildew resistance genes. Plant Cell 14:993-1003.

Tornero, P., Merritt, P., Sadanom, A., Shirasu, K., Innes, R. W., and Dangl, J. L. 2002. RAR1 and NDR1 contribute quantitatively to disease resistance in Arabidopsis, and their relative contributions are dependent on the $R$ gene assayed. Plant Cell 14:1005-1015.

Wang, Y., and Yang, M. 2006. The ARABIDOPSIS SKP1-LIKE1 (ASK1) 
protein acts predominately from leptotene to pachytene and represses homologous recombination in male meiosis. Planta 223:613-617.

Wei, N., and Deng, X. W. 2003. The COP9 signalosome. Annu. Rev. Cell Dev. Biol. 19:261-286.

Xiong, L., and Yang, Y. 2003. Disease resistance and abiotic stress tolerance in rice are inversely modulated by an abscisic acid-inducible mitogen-activated protein kinase. Plant Cell 15:745-759.

Yamamoto, T., Kimura, S., Mori, Y., Uchiyama, Y., Ishibashi, T. Hashimoto, J., and Sakaguchi, K. 2003. Interaction between proliferating cell nuclear antigen and JUN-activation-domain-binding protein 1 in the meristem of rice, Oryza sativa L. Planta 217:175-183.

Yuan, Y., Zhong, S., Li, Q., Zhu, Z., Lou, Y., Wang, L., Wang, J., Li, Q., Wang, M., Yang, D., and He, Z. 2007. Functional analysis of rice
$N P R 1$-like genes reveals that $O s N P R 1 / N H 1$ is the rice orthologue conferring disease resistance with enhanced herbivore susceptibility. Plant Biotechnol. J. 5:313-324.

Zhang, Y., Dorey, S., Swiderski, M., and Jones, J. D. 2004. Expression of RPS4 in tobacco induces an AvrRps4-independent HR that requires EDS1, SGT1 and HSP90. Plant J. 40:213-224.

Zhuang, X., Jiang, J., Li, J., Ma, Q., Xu, Y., Xue, Y., Xu, Z., and Chong, K. 2006. Over-expression of OsAGAP, an ARF-GAP, interferes with auxin influx, vesicle trafficking and root development. Plant J. 48: 581-591.

Zipfel, C., Robatzek, S., Navarro, L., Oakeley, E., Jones, J.D.G., Felix, G. and Boller, T. 2004. Bacterial disease resistance in Arabidopsis through flagellin perception. Nature 428:764-767. 